\title{
Allergy, obesity, microbiota, and fecal transplantation: what do they mean to our health?
}

\author{
L. Karla Arruda, MD, PhD1, Cristina Miuki Abe Jacob, MD, PhD²
}

In January 2013, a very interesting and provocative article was published reporting the use of fecal transplantation in treating patients with diarrhea caused by recurrent Clostridium difficile infection. ${ }^{1}$ In this study, carried out in the Netherlands, van Nood and colleagues infused healthy donor's feces into the duodenum of $C$. difficile infected patients, through a nasoduodenal tube, and compared the results obtained with this unconventional form of therapy, with the standard treatment consisting of the use of Vancomycin, combined or not with bowel lavage. Among the 16 patients of the fecal infusion group, 13 (81\%) were cured following the first infusion, and 2 out of 3 patients who received a second infusion from a different donor, no longer presented symptoms. The efficacy of the fecal transplant (94\%) was greater than that of the treatment with Vancomycin alone (31\%) or with bowel lavage (23\%). ${ }^{1}$ The technology, used for the first time in the late 50 's, ${ }^{2}$ resurfaced and is being used in Brazil: on March 30th, 2013, the newspaper "O Estado de São Paulo" commented the article by van Nood and colleagues, and reported the successful application of a variation of this form of treatment on two patients from the Albert Einstein Hospital in São Paulo, using fresh feces from donors, which were endoscopically infused into the jejunum of patients with chronic diarrhea.

A question arises: what is the relationship between fecal transplantation and allergy? It all sums up into one word: microbiota! Microbiome research has grown surprisingly, thanks to the development of techniques to identify bacterial $16 \mathrm{~S}$ ribosomal RNA in complex biological mixtures. The results of these studies have alerted us about the fundamental role of the composition and diversity of the microbiota, not only in the intestines, but also in skin and respiratory tract, and their role in maintenance of health and in the origin of metabolic, neoplastic and inflammatory diseases, including allergic illnesses.

Evidence indicates that reduced biodiversity and changes in the composition of the intestinal and skin flora are associated to various inflammatory conditions, including asthma, allergic and inflammatory bowel diseases, type 1 diabetes and obesity. Modifications of the natural microbiota, associated to less exposure to microbes, which are characteristics of the urban lifestyle in affluent countries, seem to be a risk factor for loss of immune regulatory mechanisms, resulting in loss of immunologic tolerance. The risk may be even greater when these factors are associated to lack of physical activity and/or a diet poor in fresh fruits and vegetables. Interestingly, the microbiota is variable and undergoes temporal fluctuations during the first years of life. Afterwards, equilibrium is attained, remaining stable all through life. Therefore, environmental exposures during the first years of life and diet are fundamental decisive factors for the adult intestinal microbiota.

\author{
1 Department of Medicine, School of \\ Medicine of Ribeirão Preto, University of \\ São Paulo (FMRP-USP), Ribeirão Preto, \\ $\mathrm{SP}$, Brazil. Editor-in-Chief of BJAI. \\ 2 Department of Pediatrics, School of \\ Medicine of the University of São Paulo \\ (FMUSP), São Paulo, SP, Brazil. Associate \\ Editor of BJAl.
}


In this edition of the Brazilian Journal of Allergy and Immunology (BJAI), Professor Tari Haahtela, one of the International Associated Editors of the journal, shares, in a review article, the most recent concepts about microbiota and allergy development. ${ }^{3}$ Professor Haahtela's studies with teenagers from the Karelia region, northern Finland, have shown that the skin of healthy adolescents had a greater diversity of a group of bacteria, namely the gamaproteobacteria, when compared to adolescents who were sensitized to inhalant allergens. Furthermore, among the healthy teenagers, the amount of the gamaproteobacteria Acinetobacter in the skin was positively associated to the serum levels of interleukin 10 , which has anti-inflammatory properties. A recent document from the World Allergy Organization(WAO), by Haahtela and colleagues, ${ }^{4}$ better elaborates the theory of biodiversity and allergic illnesses (online free access: http://www.waojournal.org/content/6/1/3).

Also in this issue of BJAI, Wandalsen and colleagues ${ }^{5}$ report surprising data which may be analyzed in light of the theory of biodiversity loss. In a study involving more than 9,000 infants from 5 Brazilian cities (São Paulo, Recife, Curitiba, Belém and Cuiabá), the authors have shown that accelerated weight gain was observed in $55.7 \%$ of the infants, and excessive weight gain was found in $20.8 \%$. Both events were associated to more severe forms of wheezing, while the group showing excessive weight gain was associated to medical diagnosis of asthma, regardless presence of maternal breast-feeding. These data alert us for a possible epidemic of infant obesity in our country, which could be associated to the development of asthma or even to the loss of its control. We could hypothesize that alterations in the diet and the intestinal microbiota are risk factors for obesity and more severe forms of wheezing. In keeping with these findings, the EISL (Estudio Internacional de Sibilancias en Lactantes) study, which evaluated more than 30,000 children between 12 and 15 months of age, revealed that the prevalence of recurrent wheezing was higher among Latin-american infants when compared to European babies (mean values of $21.4 \%$ and $15.0 \%$, respectively), and that morbidity from wheezing was higher in Latin America. ${ }^{5}$ This serves as a warning to all of us, as Allergists and Immunologists. We can't remain indifferent facing this intriguing reality!

Another piece of evidence regarding the potential role of the microbiota comes from a study by Oliveira and colleagues, ${ }^{6}$ also published in this issue of BJAI. When analyzing results of skin prick tests conducted on 1,514 patients with clinical symptoms suggestive of respiratory allergy in the city of Aracaju, Sergipe, the authors found a significant lower frequency of cutaneous reactivity to mites, cat, dog and cockroach Blattella germanica, among patients cared at the public health system (presumably less privileged economically), when compared to patients who were treated at private clinics. These results serve as base for future studies in our county evaluating the role of the intestinal and/or cutaneous microbiota, as in Finland, as important determinants of allergic sensitization.

Evidently, fecal transplanting is notan acceptable form of routine treatment for patients with allergic diseases. However, in the study of van Nood and colleagues, ${ }^{1}$ the authors showed that the fecal microbiota of patients with $C$. difficile infection had reduced biodiversity, and that the infusion of donor feces resulted in significant increase of biodiversity within the two weeks following transplantation, reaching levels indistinguishable from those observed in healthy donors. Furthermore, a significant increase of various types of bacteria, including the Bacteroidetes and Firmicutes species, abundant components of the healthy intestinal flora, was observed after the fecal transplant. These observations point to the possibility of new immunomodulatory therapies, including the use of probiotics and the administration of culture-obtained bacteria of defined composition. Until strategies based on the new theories are validated, it is up to us to continue emphasizing maternal breastfeeding, healthy diet, exposure to natural environments and physical activity, in addition to avoiding active or passive smoking. These measures could certainly bring benefits to our patients' health.

\section{REFERENCES}

1. van Nood E, Vrieze A, Nieuwdorp M, Fuentes S, Zoetendal EG, de Vos WM, et al. Duodenal infusion of donor feces for recurrent Clostridium difficile. N Engl J Med. 2013;368:407-15.

2. Kelly CP. Fecal microbiota transplantation - an old therapy comes of age. N Engl J Med. 2013;368:474-5.

3. Haahtela T, von Hertzen L, Hanski I. Biodiversity hypothesis explaining the rise of chronic inflammatory disorders - allergy and asthma among them - in urbanized populations? Braz J Allergy Immunol. 2013;1:5-7.

4. Haahtela et al. The biodiversity hypothesis and allergic disease: World Allergy Organization position statement. World Allergy Organization J. $2013 ; 6: 3$

5. Wandalsen GF, Borges LV, Barroso N, Navarro ACP, Suano F, Prestes EX, et al. Associação entre o ganho de peso e a prevalência e gravidade de sibilância e asma no primeiro ano de vida. Braz J Allergy Immunol. 2013;1:39-44.

6. Garcia-Marcos L, Mallol J, Solé D, Brand PL. EISL Study Group. International study of wheezing in infants: risk factors in affluent and non-affluent countries during the firstyear of life. Pediatr Allergy Immunol. 2010;21:878-88.

7. Oliveira AM, Melo EV, Nunes GA, Franco JM, Santos MA, Simões SM. Sensibilização a aeroalérgenos em pacientes com suspeita de alergia respiratória atendidos na rede pública e privada no município de Aracaju. Braz J Allergy Immunol. 2013;1:45-50. 